\title{
Propuesta de implementación de la metodología BIM 5D para obras de cimentaciones industriales en la Planta de Oxígeno de Arauco
}

\section{BIM 5D methodology implementation proposal for industrial foundation works in the Oxygen Plant in Arauco}

Fecha de entrega: 6 de septiembre 2021

Fecha de aceptación: 15 de noviembre 2021

\section{Guillermo Bustamante, Joaquín Ochoa y Felipe González}

Departamento de Ingeniería Civil, Universidad Católica de la Santísima Concepción, Alonso de Ribera 2850, Casilla 297, Concepción, Chile, gbustamante@ucsc.cl,jochoa@ing.ucsc.cl,fgonzalez@ucsc.cl

Debido al crecimiento de la industria de arquitectura, ingeniería y construcción (AEC), es primordial la actualización de las metodologías de trabajo sobre todo en la etapa de diseño y construcción, ya que aqui es en donde se concentra el costo del proyecto. El objetivo principal es la gestión de información para la prevención de errores y correcta ejecución del proyecto. El presente documento propone una estrategia para la implementación de la metodología BIM en 5D, incluyendo un modelo $3 D$, tiempo y costo, en una empresa de construcción industrial, especificamente en las obras civiles. Dicha estrategia fue diseñada en base al proyecto Planta de Oxígeno, de modo de establecer los pasos de la metodología. Para luego aplicar la metodología BIM en el proyecto, evidenciando los beneficios en costos de las actividades y estimando el costo de implementación BIM.

Palabras clave: gestión de la información, metodología, BIM 5D, obras civiles, costos
Due to the growth of the architecture, engineering and construction industry (AEC), it is essential to update the work methodologies, especially in the design and construction stage, since this is where the cost of the project is concentrated. The main objective is the management of information for the prevention of errors and the correct execution of the project. This document proposes a strategy for the implementation of the BIM methodology in 5D, including a 3D model, time and cost, in an industrial construction company, specifically in civil works. This strategy was designed on the basis of the Oxygen Plant project, in order to establish the steps of the methodology. To then apply the BIM methodology in the project, evidencing the cost benefits of the activities and estimating the cost of BIM implementation.

Keywords: management of information, methodology, BIM 5D, civil works, costs

\section{Introducción}

Debido a la alta demanda en la construcción, hoy en día la industria AEC (arquitecture, engineering \& construction) ha debido innovar y actualizarse a las tecnologías que actualmente forman una parte primordial en el estudio de proyectos, las empresas constructoras han debido mejorar sus procesos para competir y adaptarse a la evolución tecnológica del área (Pape y Nazer, 2021). En el rubro de la construcción, la gran barrera a superar es el manejo de importantes cantidades de información por proyecto, tanto en su etapa de diseño, constructiva y productiva (Aliaga, 2012). Es por esto que la metodología BIM (Building Information Modeling), ha tomado un rol fundamental en esta evolución tecnológica. BIM es una representación digital de las características físicas y funciones del proyecto, BIM se refiere al proceso y tecnología usados para crear el modelo (AIA, 2013; Eastman et al., 2018). La metodología BIM busca enlazar la información del proyecto, proporcionando una visión para ayudar a planificar, diseñar, construir y gestionar edificios e infraestructuras, siendo más eficiente que las metodologías anteriores, pues a diferencia de otras BIM se basa en Lean management (autodesk.com). En general, la aplicación de BIM 5D en construcción no ha sido ampliamente adoptada debido a la reticencia a cambiar procedimientos establecidos en el tiempo, entre otras causas (Forgues et al., 
2012; Hasan y Rasheed, 2019; Kim et al., 2016; Stanley y Thurnell, 2014).

En Chile, según PMG bussines improvement 2018 (programa de mejoramiento de la gestión pmgchile.com), un $69 \%$ de las empresas del área de construcción declaran no realizar proyectos en BIM, además solo un $10 \%$ de las empresas constructoras declaran haber utilizado BIM en alguna de sus etapas. Vale señalar que la mayor parte de las empresas que utilizan BIM, se encuentran en el área de diseño e ingeniería, denotando un déficit en las áreas de construcción y operación de las estructuras. En el año 2016, bajo la tutela de la CORFO (2015) y su programa Construye 2025, se crea el Plan BIM, el cual busca incrementar la productividad y sustentabilidad de la industria de la construcción. Además, el programa establece fechas límites para la utilización de la metodología tanto para el área pública como privada, siendo el año 2020 y 2025, correspondientemente.

Según los datos recolectados por PMG 2018, en la región del Biobío solo un $27 \%$ de los profesionales declara tener algún conocimiento de BIM. Es por esto que urge la necesidad de actualizar los procesos internos de las empresas para preparar, capacitar e incorporar personal para las etapas de diseño y construcción dentro de un proyecto (PMG, 2018).

El presente trabajo busca establecer una estrategia para implementar la metodología BIM en el área de la construcción industrial, aplicado en el proyecto: Construcción de obras civiles de una planta de oxígeno, realizado por una empresa constructora. Al aplicarse la metodología a este proyecto en particular, se busca también que se pueda replicar en cualquier otro proyecto industrial de obras civiles, específicamente cimentaciones, realizados por la empresa a futuro. Además, establecer parámetros comparativos de los beneficios para la empresa al momento de afrontar una licitación de proyecto $\mathrm{y}$ posterior ejecución.

En este trabajo se propone una metodología de estudio de propuestas para obras civiles industriales, utilizando el sistema BIM 5D, con el propósito de identificar los beneficios de su uso, para disminuir costos en modificaciones o errores de presupuesto. Para ello, se necesita identificar softwares a implementar en el modelo $3 \mathrm{D}$, establecer parámetros para la planificación, dada la eficiencia de los recursos pertinente a cada partida, obtener el modelo BIM mediante la vinculación del modelo 3D con la planificación y comparar la metodología de la empresa y BIM, con el fin de proponer soluciones para la eficiencia.

\section{Descripción de la obra}

La obra a desarrollar se trata de las fundaciones para equipos de una planta de oxígeno, cuyo producto final es oxígeno líquido, el cual es suministrado a la planta de Arauco Horcones, para su proceso productivo en la ampliación de dicha planta (Figura 1). Al tratarse de una planta industrial, las fundaciones son de gran envergadura, tanto por la resistencia, la altura de los elementos posteriormente montados, por las vibraciones que produce el funcionamiento de la planta, la cual contempla equipos como sopladores de gran tamaño, motores, transformadores, estanques de almacenamiento, equipos criogénicos, skid de procesamiento, entre otros. Dicho proyecto consta de 5 fundaciones, las cuales en el interior poseen fundaciones especiales dependiendo del requerimiento de cada equipo. Dada la situación sanitaria del país se debe considerar el aumento de tiempo y gastos que implica la correcta y responsable ejecución de la obra, las cuales afectan el tiempo y costo de la obra. Este trabajo está desarrollado en la época de pandemia y hay costos que en condiciones normales no son exactamente como los indicados en este trabajo.

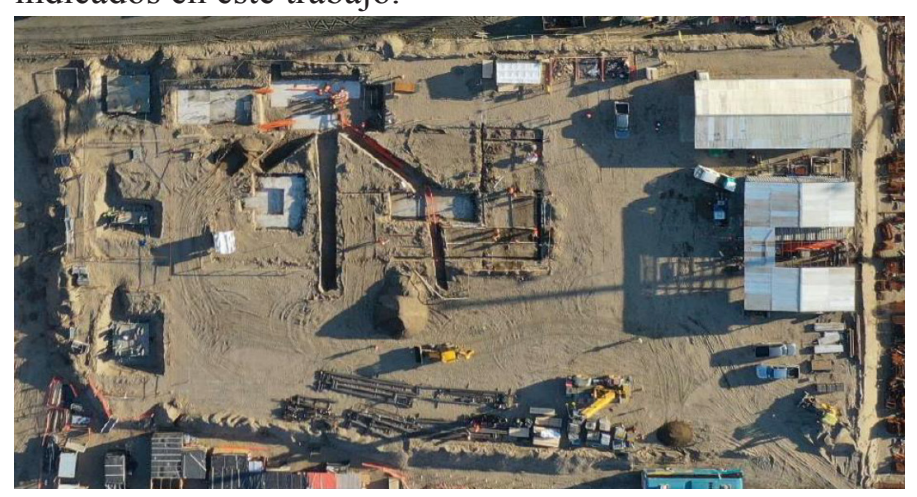

Figura 1: Foto aérea de la plataforma de la obra

\section{Metodología BIM}

BIM, por sus siglas en inglés (Building Information Model), se define como una metodología de trabajo colaborativo para la concepción y gestión de proyectos de edificación y obras civiles (buildingsmart.org). A su vez, BIM es un proceso para crear y administrar información sobre un proyecto de construcción a lo largo del ciclo de 
vida del proyecto (nationalbimlibrary.com). Se entiende BIM como una metodología de trabajo que va más allá de la modelación del proyecto en $3 \mathrm{D}$, siendo una forma de trabajo interdisciplinaria y colaborativa. Por lo demás, BIM permite gestionar el proyecto en la fase de construcción, donde es de vital importancia que las especialidades trabajen en conjunto, de modo de tener más claridad del proyecto y anticipando los posibles errores, los cuales inducen a atrasos dentro del proyecto, impactando en los costos proyectados y en la planificación (Eastman et al., 2018).

El uso de metodología BIM se ha vuelto fundamental para el desarrollo de la industria AEC, por lo que ha surgido la urgencia de implementar normativas para el control de BIM (ISO 19650-1, 2018; ISO 19650-2, 2018).

En la metodología BIM, se habla de las dimensiones y las fases de BIM. Se considera que las dimensiones son 7 , teniendo en cuenta que todo proyecto nace de una idea y termina con el fin de la vida útil de la infraestructura (buildingsmart.es). Por otra parte, según Montilla (2017), las fases de BIM pueden ser separadas en:

- Fase de diseño: Se define el modelo gráfico, planificación y estimaciones de costos, de modo de realizar los análisis y la colaboración entre especialidades.

- Fase de ejecución: Es el desarrollo de la programación, la documentación gráfica del proyecto y de todo el periodo de construcción incluyendo la puesta en marcha. Desde este punto se habla del modelo as-built.

- Fase de operación: Se incluyen las operaciones después de la entrega, como son la gestión de activos, mantenimiento y fin del ciclo de vida del edificio.

Además, en la fase de diseño es donde las especialidades se coordinan, por lo cual divide la fase en las especialidades que influyen en el modelo virtual, de las cuales se obtendrán 3 modelos: arquitectónico, estructural y MEP (mecánico, eléctrico y plomería).

Según Plan BIM Chile 2020 (planbim.cl), las ventajas que se generan al utilizar la metodología BIM, desde su fase de diseño hasta su fase de operación, son: a) mejora la administración de datos e información compleja de proyectos, b) los modelos 3D facilitan la comprensión y expectativas del cliente, c) optimiza los flujos de trabajo, fomentando el trabajo interdisciplinario, d) permite solucionar problemas de manera anticipada, e) optimiza la planificación de costos y plazos del proyecto y f) permite mejorar la sustentabilidad y eficiencia del proyecto, a través de su modelación.

Si bien los costos de la obra se pueden ver reducidos por los beneficios de BIM, la metodología tiene costos asociados para llevar a cabo su correcto funcionamiento, tales como capacitación y contratación de personal. Además de la disposición del material necesario para trabajar como hardware y software, donde se deben considerar los costos de equipamiento, estandarización y licencias asociadas. Sin embargo, los costos de implementación de la metodología tienden a cero en el transcurso del tiempo y los beneficios serán mayores, dependiendo de la magnitud del proyecto (CORFO, 2015).

\section{Metodología}

Este trabajo abordará la estrategia de adaptación de la metodología BIM para el estudio de propuestas y licitaciones, implementada en la empresa con el fin de optimizar el estudio de la licitación y evitar errores en el desarrollo del proyecto. Para esto se deben establecer los parámetros y acciones a tomar en el desarrollo de información BIM.

Se establecerá una estrategia, donde se estipularán roles e identificarán los beneficios que surgen al utilizar la metodología, basado en la forma de trabajo actualmente aplicada por la empresa. Se realizará un procedimiento de gestión de la información, identificando los puntos primordiales dentro del estudio para generar los productos BIM, pudiendo identificar y ejemplificar las recomendaciones de los pasos a seguir. Para la metodología se utilizarán los softwares más utilizados a nivel local, siendo estos MS PROJESCT (planificación y costo), AUTOCAD (antecedentes 2D), REVIT (modelo 3D) y NAVISWORKS (vinculación y simulación).

\section{Estrategia de la metodología}

La principal función de la metodología es la gestión de información del proyecto. Es por eso que para la aplicación es necesario identificar al personal que será imprescindible para el desarrollo de las actividades del proyecto BIM. Se debe aplicar la metodología desde la licitación, 
distribuyendo la información a las diferentes especialidades involucradas, para luego crear la planificación y costo de cada actividad a realizar. El encargado del proyecto será el coordinador BIM, el cual tendrá como función la intercomunicación entre los diferentes participantes del proyecto. En el caso de las licitaciones para construcción, la fase de diseño es suministrada por el cliente, el cual previamente desarrolla la ingeniería con que se llevará a cabo el proceso de licitación.

Para la postulación se entregarán por parte del mandante las bases administrativas, especificaciones técnicas y los planos, necesarios para la comprensión y estudio del proyecto. Este será el punto de partida de la metodología a aplicar, debiendo llevar los planos entregados en 2D (AutoCAD) a un modelo 3D, el cual será realizado mediante Revit (Autodesk). Dicho modelo deberá ser enlazado a la planificación, la que tendrá los suministros necesarios por partida y los tiempos necesarios. La estimación de personal, se deberá realizar según el rendimiento, ajustándose a los plazos entregados por el mandante, si éste los especifica. Uno de los grandes beneficios de utilizar la metodología $\mathrm{BIM}$, es la fácil interpretación del proyecto, ya que además de poder generar un modelo 3D y poder presentar la maqueta digital ante cualquier duda, se podrá realizar una simulación del proyecto, según la planificación vinculada, la que también incluye los costos asociados a cada actividad, por lo que se vería el avance del costo económico en cualquier punto del proyecto (Trejo, 2018).

\section{Antecedentes del proyecto}

Para la licitación del presente proyecto, el mandante entregó como antecedentes de la obra las bases administrativas, EETT y planos de construcción. Dentro de dichos planos y EETT, se encuentran la ingeniería básica, dentro de la cual se destacan las actividades de obras civiles como: fundaciones especiales, losas estructurales, edificios de hormigón armado, pernos embebidos, muros cortafuegos, cierro perimetral de la planta, drenaje de aguas lluvias y normalización del área.

La ingeniería de detalle fue subcontratada por la empresa, la cual corresponde a la enfierradura de todas las fundaciones, cuyo suministro y doblado fue contratado a una empresa externa. El proyecto como se mencionó anteriormente se trata de una planta de oxígeno líquido, donde el mandante es quien suministra los equipos provenientes del extranjero, por lo que se debe tener en cuenta que toda consulta sobre el proyecto, se debe hacer mediante una Solicitud de información (SDI) a LINDE GROUP, los cuales tendrán que realizar aclaraciones con ingeniería de diseño o solicitar la solución en terreno. El proceso requiere de un tiempo de respuesta no menor, lo cual podría significar un atraso en la planificación de la obra.

La obra se encuentra en la Planta Arauco Horcones, ubicada en la cuidad de Arauco en el borde costero (Figura 1). El suelo de fundación es arena de playa. Los trabajos de compactación y mejoramiento de suelo fueron realizados por una empresa externa especializada, previo a la entrega del terreno. Los antecedentes para la construcción de las fundaciones contempladas en el proyecto, constan de 31 planos de ingeniería básica más 5 planos de ingeniería de detalle y sus respectivas especificaciones técnicas.

\section{Roles en BIM}

Los roles dentro de BIM son clasificados en 5 roles, los cuales pueden ser compuestos por más de una persona o una persona ejercer más de un rol. Se debe tener en cuenta que un rol, no es un cargo dentro del proyecto, sino responsabilidades para ejercer las acciones necesarias (Plan BIM, 2017).

- Dirección: fomenta la utilización de BIM en la empresa, de acuerdo a sus necesidades y toma de decisiones en proyectos e inversiones.

- Revisión: verifica la información y datos de los modelos a desarrollar.

- Modelación: desarrolla los modelos según la especialidad, extrayendo información técnica. Modela elementos agregando o actualizando la información.

- Coordinación: realiza la integración de la información de los diferentes participantes del proceso, previene los conflictos y asegura la correcta modelación del proyecto. Mantiene la información actualizada y es el principal punto de contacto entre modeladores y gerencia.

- Gestión: es responsable de la planificación, administración de los recursos humanos y desarrollo del proyecto. Define los modelos que deberán ser creados, la vinculación entre los modelos y la organización de la información. 
En este punto se propondrán los roles de metodología BIM para el personal de la empresa. Se designarán los participantes y cargos, de modo de identificar los participantes del proyecto. En la Tabla 1, en la primera columna se muestran los roles necesarios para BIM, en la segunda columna se muestra el personal existente en la empresa. Además, se incluyen las especialidades, control de calidad, prevención y medioambiente, quienes aportan información importante a la hora de licitaciones. En la Tabla 2 se muestra el organigrama de tareas específicas a realizar por el personal mencionado en la Tabla 1 según su rol dentro del proyecto.

Tabla 1: Asignación roles BIM para empresa

\begin{tabular}{|c|l|}
\hline Rol & \multicolumn{1}{|c|}{ Responsable(s) en Empresa } \\
\hline Dirección & Gerencia \\
\hline Revisión & $\begin{array}{l}\text { Depto. Estudio de Propuestas } \\
\text { Aseguramiento de Calidad }\end{array}$ \\
\hline Modelación & $\begin{array}{l}\text { Depto. Estudio de Propuestas } \\
\text { Oficina Técnica de Obra }\end{array}$ \\
\hline Coordinación & $\begin{array}{l}\text { Depto. Estudio de Propuestas } \\
\text { Oficina Técnica de Obra }\end{array}$ \\
\hline Gestión & $\begin{array}{l}\text { Depto. Estudio de Propuestas } \\
\text { Depto. Recursos Humanos } \\
\text { Depto. Adquisiciones } \\
\text { Administrador de Contrato } \\
\text { Oficina Técnica de Obra } \\
\text { Prevención de Riesgos }\end{array}$ \\
\hline
\end{tabular}

\section{Dotación y suministro}

El cálculo de la dotación de personal, para este caso, será responsabilidad de Gestión BIM, específicamente del Depto. de Estudio de Propuestas. Sin embargo, la contratación será responsabilidad del Depto. de Recursos Humanos. Se considerará solo el personal directo de obra, dado que la planificación está basada en el costo por actividad, así se podrá obtener el costo directo de cada tarea a realizar en el proyecto. Además, los recursos serán ingresados en la planificación, por lo que también se podrá identificar los recursos necesarios por actividad. El suministro de los materiales y herramientas de menor envergadura es solicitado por el administrador de contrato. Dicha solicitud, es recepcionada por el Depto. de Adquisiciones, el cual gestionará la compra y despacho, con previa aprobación de gerencia. No así los materiales de gran envergadura los cuales deben ser parte de la planificación, por lo que el administrador de contrato realizará su compra directamente con la empresa externa, con previa aprobación del ingeniero planificador
Tabla 2: Responsabilidades según rol en BIM

\begin{tabular}{|c|c|c|}
\hline Departamento & Rol & Responsabilidad \\
\hline Gerencia & Dirección & Toma de decisiones \\
\hline \multirow{4}{*}{$\begin{array}{l}\text { Estudio de } \\
\text { propuestas }\end{array}$} & Gestión & $\begin{array}{l}\text { Cálculo de Recursos } \\
\text { Planificación } \\
\text { Determinación de modelos a } \\
\text { generar } \\
\text { Costos generales de obra }\end{array}$ \\
\hline & Modelación & $\begin{array}{l}\text { Modelo 3D } \\
\text { Procesamiento información } \\
\text { antecedentes }\end{array}$ \\
\hline & Coordinación & $\begin{array}{l}\text { Vinculación, Procesamiento } \\
\text { de información y Simulación } \\
\text { de Proyectos }\end{array}$ \\
\hline & Revisión & $\begin{array}{l}\text { Revisión de materiales y } \\
\text { técnicas constructivas, según } \\
\text { especificaciones }\end{array}$ \\
\hline Adquisiciones & Gestión & $\begin{array}{l}\text { Cotización y Adquisición de } \\
\text { Materiales } \\
\text { Estipulación costos Recursos }\end{array}$ \\
\hline Recursos Humanos & Gestión & Enrolamiento Personal \\
\hline \multirow{2}{*}{$\begin{array}{l}\text { Aseguramiento de } \\
\text { Calidad }\end{array}$} & Revisión & $\begin{array}{l}\text { Comprobación de Materiali- } \\
\text { dad y forma, según normativa }\end{array}$ \\
\hline & Gestión & $\begin{array}{l}\text { Generación de documentación } \\
\text { sistema de gestión integrado }\end{array}$ \\
\hline $\begin{array}{l}\text { Prevención de } \\
\text { Riesgo }\end{array}$ & Gestión & $\begin{array}{l}\text { Estándares y capacitación de } \\
\text { obra }\end{array}$ \\
\hline \multirow{2}{*}{$\begin{array}{l}\text { Administrador de } \\
\text { Contrato }\end{array}$} & Coordinación & $\begin{array}{l}\text { Procesamiento información } \\
\text { de obra }\end{array}$ \\
\hline & Gestión & $\begin{array}{l}\text { Estipulación de cronograma y } \\
\text { reuniones }\end{array}$ \\
\hline \multirow{4}{*}{ Oficina Técnica } & Modelación & Actualización de modelo 3D \\
\hline & Gestión & $\begin{array}{l}\text { Actualización de Planifica- } \\
\text { ción }\end{array}$ \\
\hline & Revisión & $\begin{array}{l}\text { Revisisón de técnicas y } \\
\text { materiales en terreno, según } \\
\text { estándares }\end{array}$ \\
\hline & Coordinación & $\begin{array}{l}\text { Vinculación y resolución de } \\
\text { información aportada de obra }\end{array}$ \\
\hline
\end{tabular}

Tabla 3: Personal directo e indirecto de obra.

\begin{tabular}{|l|l|c|}
\hline Personal & \multicolumn{1}{|c|}{ Cargo } & Cantidad \\
\hline \multirow{4}{*}{ Indirecto } & Administrador de Contrato & 1 \\
\cline { 2 - 3 } & Planificador de Obra & 1 \\
\cline { 2 - 3 } & Aseguramiento Calidad & 2 \\
\cline { 2 - 3 } & Prevención de Riesgo & 2 \\
\cline { 2 - 3 } & Administrativo & 1 \\
\hline \multirow{5}{*}{ Directos } & Supervisor de obra & 1 \\
\cline { 2 - 3 } & Topógrafo & 3 \\
\cline { 2 - 3 } & Capataz & 15 \\
\cline { 2 - 3 } & Maestro Primera & 14 \\
\cline { 2 - 3 } & Maestro Segunda & 15 \\
\cline { 2 - 3 } & Operadores & \\
\cline { 2 - 3 } & Enfierradores & 14 \\
\hline
\end{tabular}


de obra y gerencia. El personal indirecto necesario para llevar a cabo la obra se muestra en la Tabla 3, junto con el personal directo de la obra, los cuales se subdividen según la especialidad del personal. El personal Directo en este informe, será considerado como todo aquel que no pertenezca a la Oficina Técnica de obra.

\section{Traspaso de 2D a 3D}

El traspaso de información de los planos al modelo 3D es la primera y principal tarea a desarrollar en la metodología, ya que aquí se crea el modelo en base al cual, se calcularán los recursos y tiempos del proyecto. Para efecto de modelación del proyecto, se utiliza el software de modelación REVIT, debido que a nivel local (Chile y Sudamérica), el desarrollador más utilizado es Autodesk. El modelo será creado a partir de la información de antecedentes entregada por el mandante en la licitación. El desarrollo de dicho modelo será responsabilidad del Depto. de Estudio de Propuesta, como rol de Modelación BIM. Para el traspaso de información al modelo 3D se utilizarán los planos entregados por el mandante. Así se identificarán la posición y cota de los elementos a construir. Al tener los elementos referenciados, se crean sobreponiendo el elemento al plano de referencia. A modo de aclaración se puede crear el modelo sin la utilización de las referencias del modelo 2D, solo con la lectura manual. Para este caso se utilizarán las referencias.

\section{Modelo 3D}

El modelo 3D estará a cargo de la oficina de estudio de propuesta, bajo supervisión del coordinador BIM, se recomienda la inclusión de un proyectista o dibujante, que sea quien traspase la información, el cual estará a cargo del jefe de estudio de propuesta o coordinador BIM. El modelo debe incluir las especialidades que conciernen a la obra para este caso la especialidad civil y arquitectónica, cuyos parámetros se muestran a continuación.

\section{Modelo arquitectónico (geometría)}

Para el modelo arquitectónico es necesario referenciar la geometría de las fundaciones, establecer la posición de postes de iluminación, delimitar el cierro perimetral, tener en claro las cotas de sello de fundación, terreno natural, estabilizado y tope de hormigón. Todos los materiales utilizados en el proyecto, deben ser modelados como tal en el programa Revit.

\section{Modelo ingenieril (refuerzo estructural)}

Una vez creados todos los elementos de fundaciones, losas y edificios en el modelo arquitectónico, se debe añadir la enfierradura pertinente a cada elemento. Para la fase de construcción, no es necesario realizar el modelo analítico. En la Figura 2 se muestra el modelo 3D del proyecto, en el cual se observa la geometría, materialidad y ubicación de los diferentes elementos anteriormente mencionados. Por otra parte, en la Figura 3, se muestra la armadura de refuerzo de los distintos elementos.

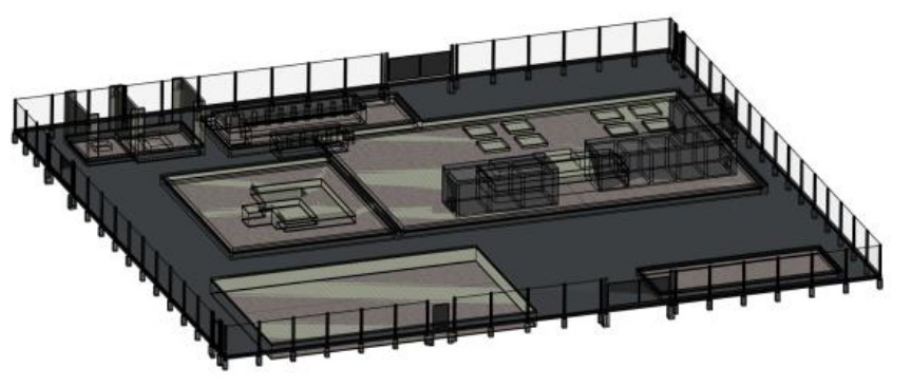

Figura 2: Vista 3D modelo arquitectónico, software Revit.

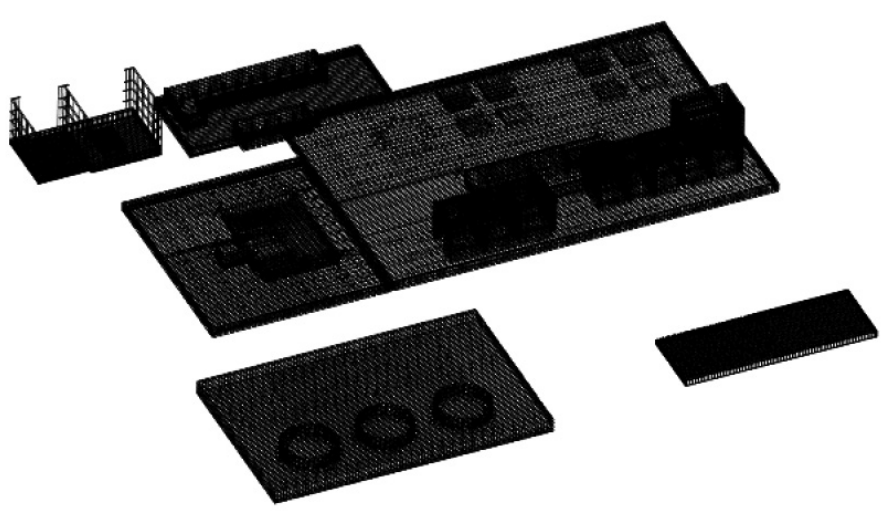

Figura 3: Vista 3D modelo ingenieril (refuerzo), software Revit

\section{Cubicación}

La cubicación es uno de los beneficios al utilizar la metodología BIM, ya que, al modelar el proyecto, el programa Revit es capaz de realizar la cubicación de todos los materiales empleados en la obra. La cubicación es responsabilidad de la oficina de estudio de propuesta, bajo su rol de gestión BIM. La planilla mostrada en la Figura 4, es una planilla tipo de la información extraída desde el software Revit, mediante las tablas de planificación. Éstas son construidas con los materiales ingresados en la etapa de modelación. Las planillas de materiales deben ser extraídas por grupos o tipo de material, por lo que existirá más de una planilla de materiales para el proyecto. Una 
vez obtenida la planilla requerida, es exportada al software Excel, como se muestra en la Figura 5.

\begin{tabular}{|c|c|c|c|c|}
\hline \multicolumn{5}{|c|}{ Cubicacion Cimentacion 1} \\
\hline Tipo & Familia & Volumen & $\begin{array}{c}\text { Recue } \\
\text { nto }\end{array}$ & Material estructural \\
\hline Cimientos acceso peatonal & M_Zapata-Rectangular & $0.43 \mathrm{~m}^{3}$ & 6 & Hormigon, Moldeado in situ, gris \\
\hline Cimientos acceso vehicular & M_Zapata-Rectangular & $1.20 \mathrm{~m}^{3}$ & 6 & Hormigón, Moldeado in situ, gris \\
\hline Cimientos Cerco & M_Zapata-Rectangular & $4.28 \mathrm{~m}^{3}$ & 68 & Hormigón, Moldeado in situ, gris \\
\hline Emplantillado & Foundation Slab & $53.05 \mathrm{~m}^{3}$ & 17 & Hormigon, moldeado in situ \\
\hline Fundacion Compresor $1.5 \mathrm{~m}$ & Foundation Slab & $21.56 \mathrm{~m}^{3}$ & 1 & Hormigón, Moldeado in situ, gris \\
\hline Fundacion Compresor $1 \mathrm{~m}$ & Foundation Slab & $27.96 \mathrm{~m}^{3}$ & 1 & Hormigón, Moldeado in situ, gris \\
\hline Fundacion pernos & Foundation Slab & $7.10 \mathrm{~m}^{3}$ & 8 & Hormigön, Moldeado in situ, gris \\
\hline Losa Almacen & Foundation Slab & $12.51 \mathrm{~m}^{3}$ & 1 & Hormigón, Moldeado in situ, gris \\
\hline Losa DRIOX & Foundation Slab & $315.90 \mathrm{~m}^{3}$ & 1 & Hormigón, Moldeado in situ, gris \\
\hline Losa SALA ELECTRICA & Foundation Slab & $22.91 \mathrm{~m}^{3}$ & 2 & Hormigón, Moldeado in situ, gris \\
\hline Losa TRANSFORMADOR 1 & Foundation Slab & $4.22 \mathrm{~m}^{3}$ & $\frac{c}{1}$ & Hormigón, Moldeado in situ, gris \\
\hline Losa TRANSFORMADOR 2 & Foundation Slab & $3.17 \mathrm{~m}^{3}$ & 1 & Hormigón, Moldeado in situ, gris \\
\hline $\begin{array}{l}\text { Losa VPSA } \\
\end{array}$ & Foundation Slab & $272.58 \mathrm{~m}^{3}$ & 2 & Hormigón, Moldeado in situ, gris \\
\hline Pedestal B.1 & M_Zapata-Rectangular & $4.72 \mathrm{~m}^{3}$ & 1 & Hormigón, Moldeado in situ, gris \\
\hline Pedestal B.2 & M_Zapata-Rectangular & $1.09 \mathrm{~m}^{3}$ & 1 & Hormigón, Moldeado in situ, gris \\
\hline Pedestal B.3 & M_Zapata-Rectangular & $4.95 \mathrm{~m}^{3}$ & 1 & Hormigón, Moldeado in situ, gris \\
\hline Pedestal Blower & M_Zapata-Rectangular & $55.43 \mathrm{~m}^{3}$ & 1 & Hormigón, Moldeado in situ, gris \\
\hline Pedestal transformador $6,9 \mathrm{kv}$ & M_Zapata-Rectangular & $0.46 \mathrm{~m}^{3}$ & 1 & Hormigón, Moldeado in situ, gris \\
\hline Pedestal transformador $33 \mathrm{kV}$ & M Zapata-Rectangular & $2.29 \mathrm{~m}^{3}$ & 1 & Hormigón, Moldeado in situ, gris \\
\hline
\end{tabular}

Figura 4: Planilla de planificación/cantidad, software Revit

Además de la cubicación realizada por el programa, se debe realizar el levantamiento de los materiales que no fueron insertos en el modelo 3D, si existiesen. Para este caso, los pernos de anclaje embebidos no fueron proyectados, dada su baja magnitud dentro del modelo, pero sí considerados en costo y tiempo. Es aquí, donde el coordinador BIM juega un rol importante dentro de la licitación, ya que es el responsable de la recolección y vinculación de la información de las distintas especialidades, dentro del proyecto.

\section{Planificación}

La planificación es la cuarta dimensión del proyecto. Se incluye la variable tiempo al proyecto, por esto se debe realizar en base al modelo 3D, y así darle un orden lógico constructivo. Además, se aprecia con mayor facilidad la envergadura y detalles del proyecto. Se debe respetar los rendimientos estipulados por la oficina de estudio de propuesta y debe ser constantemente chequeada para controlar los tiempos por actividad, cada actividad tiene que contar con los recursos necesarios para su realización, los cuales deberán ser ingresados en el software con sus respectivas cantidades y costos. Esta información será solicitada al depto. de estudio de propuestas y el de adquisiciones, respectivamente. La Tabla 4 muestra un extracto de los recursos necesarios para la realización de las actividades descritas en la planificación. Cabe señalar

\begin{tabular}{|c|c|c|c|c|}
\hline \multicolumn{5}{|c|}{ Cubicación Cimentación } \\
\hline Tipo & Familia & Volumen & Recuento & Material estructural \\
\hline Cimientos acceso peatonal & M_Zapata-Rectangular & $0.43 \mathrm{~m}^{3}$ & 6 & Hormigón, Moldeado in situ, gris \\
\hline Cimientos acceso vehicular & M_Zapata-Rectangular & $1.20 \mathrm{~m}^{3}$ & 6 & Hormigón, Moldeado in situ, gris \\
\hline Cimientos Cerco & M_Zapata-Rectangular & $4.28 \mathrm{~m}^{3}$ & 68 & Hormigón, Moldeado in situ, gris \\
\hline Emplantillado & Foundation Slab & $53.05 \mathrm{~m}^{3}$ & 17 & Hormigón, moldeado in situ \\
\hline Fundacion Compresor $1.5 \mathrm{~m}$ & Foundation Slab & $21.56 \mathrm{~m}^{3}$ & 1 & Hormigón, Moldeado in situ, gris \\
\hline Fundacion Compresor 1m & Foundation Slab & $27.96 \mathrm{~m}^{3}$ & 1 & Hormigón, Moldeado in situ, gris \\
\hline Fundacion pernos & Foundation Slab & $7.10 \mathrm{~m}^{3}$ & 8 & Hormigón, Moldeado in situ, gris \\
\hline Losa Almacen & Foundation Slab & $12.51 \mathrm{~m}^{3}$ & 1 & Hormigón, Moldeado in situ, gris \\
\hline Losa DRIOX & Foundation Slab & $315.90 \mathrm{~m}^{3}$ & 1 & Hormigón, Moldeado in situ, gris \\
\hline Losa SALA ELECTRICA & Foundation Slab & $22.91 \mathrm{~m}^{3}$ & 2 & Hormigón, Moldeado in situ, gris \\
\hline Losa TRANSFORMADOR 1 & Foundation Slab & $4.22 \mathrm{~m}^{3}$ & 1 & Hormigón, Moldeado in situ, gris \\
\hline Losa TRANSFORMADOR 2 & Foundation Slab & $3.17 \mathrm{~m}^{3}$ & 1 & Hormigón, Moldeado in situ, gris \\
\hline Losa VPSA & Foundation Slab & $272.58 \mathrm{~m}^{3}$ & 2 & Hormigón, Moldeado in situ, gris \\
\hline Pedestal B.1 & M_Zapata-Rectangular & $4.72 \mathrm{~m}^{3}$ & 1 & Hormigón, Moldeado in situ, gris \\
\hline Pedestal B.2 & M_Zapata-Rectangular & $1.09 \mathrm{~m}^{3}$ & 1 & Hormigón, Moldeado in situ, gris \\
\hline Pedestal B.3 & M_Zapata-Rectangular & $4.95 \mathrm{~m}^{3}$ & 1 & Hormigón, Moldeado in situ, gris \\
\hline Pedestal Blower & M_Zapata-Rectangular & $55.43 \mathrm{~m}^{3}$ & 1 & Hormigón, Moldeado in situ, gris \\
\hline Pedestal transformador $6,9 \mathrm{kv}$ & M_Zapata-Rectangular & $0.46 \mathrm{~m}^{3}$ & 1 & Hormigón, Moldeado in situ, gris \\
\hline Pedestal transformador $33 \mathrm{kV}$ & M_Zapata-Rectangular & $2.29 \mathrm{~m}^{3}$ & 1 & Hormigón, Moldeado in situ, gris \\
\hline
\end{tabular}

Figura 5: Planilla de hormigones de cimentación importada desde Revit a Excel 
Tabla 4: Extracto planilla de recursos

\begin{tabular}{|c|c|c|c|c|c|}
\hline Nombre del recurso & Tipo & $\begin{array}{c}\text { Etiqueta de } \\
\text { material }\end{array}$ & Grupo & Tasa estándar & Costo/Uso \\
\hline Fierro Estriado $n^{\circ} 6$ & Material & $\mathrm{kg}$ & Armadura & $\$ 650$ & $\$ 0$ \\
\hline Fierro Estriado $n^{\circ} 8$ & Material & $\mathrm{kg}$ & Armadura & $\$ 650$ & $\$ 0$ \\
\hline Fierro Estriado $n^{\circ} 10$ & Material & $\mathrm{kg}$ & Armadura & $\$ 650$ & $\$ 0$ \\
\hline Fierro Estriado $n^{\circ} 12$ & Material & $\mathrm{kg}$ & Armadura & $\$ 650$ & $\$ 0$ \\
\hline Fierro Estriado $n^{\circ} 16$ & Material & $\mathrm{kg}$ & Armadura & $\$ 650$ & $\$ 0$ \\
\hline Fierro Estriado $n^{\circ} 18$ & Material & $\mathrm{kg}$ & Armadura & $\$ 650$ & $\$ 0$ \\
\hline Fierro Estriado $n^{\circ} 22$ & Material & $\mathrm{kg}$ & Armadura & $\$ 650$ & $\$ 0$ \\
\hline Fierro Estriado $n^{\circ} 25$ & Material & $\mathrm{kg}$ & Armadura & $\$ 650$ & $\$ 0$ \\
\hline Fierro Estriado $n^{\circ} 28$ & Material & $\mathrm{kg}$ & Armadura & $\$ 650$ & $\$ 0$ \\
\hline Alambre Negro & Material & $\mathrm{kg}$ & Armadura & $\$ 600$ & $\$ 0$ \\
\hline Retroescavadora & Trabajo & & Máquina & $\$ 24000 /$ hora & $\$ 0$ \\
\hline Grúa 20 Ton & Trabajo & & Máquina & $\$ 24000 /$ hora & $\$ 0$ \\
\hline Rodillo Vibrador & Trabajo & & Máquina & $\$ 12$ 000/hora & $\$ 0$ \\
\hline Sonda Vibradora 1 & Trabajo & & Máquina & $\$ 800 /$ hora & $\$ 0$ \\
\hline Sonda Vibradora 2 & Trabajo & & Máquina & $\$ 800 /$ hora & $\$ 0$ \\
\hline Sonda Vibradora 3 & Trabajo & & Máquina & $\$ 800 /$ hora & $\$ 0$ \\
\hline Estación Total & Trabajo & & & $\$ 1000 /$ hora & $\$ 0$ \\
\hline Laboratorio Suelo & Material & Muestra & Servicio & $\$ 10.000$ & $\$ 130.000$ \\
\hline Servicio de Bombeo Hormigón & Material & $\mathrm{m} 3$ & Servicio & $\$ 15.000$ & $\$ 0$ \\
\hline Laboratorio Hormigón & Material & Muestra & Servicio & $\$ 40.000$ & $\$ 130.000$ \\
\hline Hormigón H10 & Material & $\mathrm{m} 3$ & Material & $\$ 120.000$ & $\$ 0$ \\
\hline Hormigón H30 & Material & $\mathrm{m} 3$ & Material & $\$ 130.000$ & $\$ 0$ \\
\hline Hormigón H30-A & Material & $\mathrm{m} 3$ & Material & $\$ 140.000$ & $\$ 0$ \\
\hline Grava & Material & $\mathrm{m} 3$ & Material & $\$ 15.000$ & $\$ 0$ \\
\hline Placa Colaborante & Material & $\mathrm{m} 2$ & Material & $\$ 25.000$ & $\$ 0$ \\
\hline Pino $\operatorname{dim} 2 \times 2$ & Material & $\mathrm{m} 2$ & Material & $\$ 4.500$ & $\$ 0$ \\
\hline Pino $\operatorname{dim} 2 \times 4$ & Material & $\mathrm{m} 2$ & Material & $\$ 4.000$ & $\$ 0$ \\
\hline Placa OSB & Material & $\mathrm{m} 2$ & Material & $\$ 4.400$ & $\$ 0$ \\
\hline Fieltro & Material & $\mathrm{m} 2$ & Material & $\$ 400$ & $\$ 0$ \\
\hline Portón Vehicular & Material & Unid & Material & $\$ 1.062 .000$ & $\$ 0$ \\
\hline Portón Peatonal & Material & Unid & Material & $\$ 271.200$ & $\$ 0$ \\
\hline Malla ACMA & Material & $\mathrm{ml}$ & Material & $\$ 5.750$ & $\$ 0$ \\
\hline Postes Hormigón Prefabricado & Material & unid & Material & $\$ 150.000$ & $\$ 0$ \\
\hline
\end{tabular}

que las planillas son extraídas en formato pdf desde Project.

El depto. de estudio de propuesta es el encargado de la planificación en la fase de licitación, bajo el rol de Gestión BIM, para posteriormente hacer entrega al planificador de obra, quien hará el seguimiento y actualización de las actividades. En la fase de licitación la planificación debe ser entregada a gerencia y prevención de riesgo, para su aprobación y gestión de los estándares necesarios.

\section{Costos y productividad}

La determinación del costo directo asociado a la obra, se verá reflejado una vez que sea realizada la planificación con los recursos. Se podrá extraer la planilla de costos diferenciando los gastos de materiales, mano de obra y equipos asociados a las tareas. Los costos indirectos de la obra, serán estimados por el departamento de estudio de propuestas e incluidos en la licitación. La metodología ofrece una gran ventaja a la hora de identificar los costos 


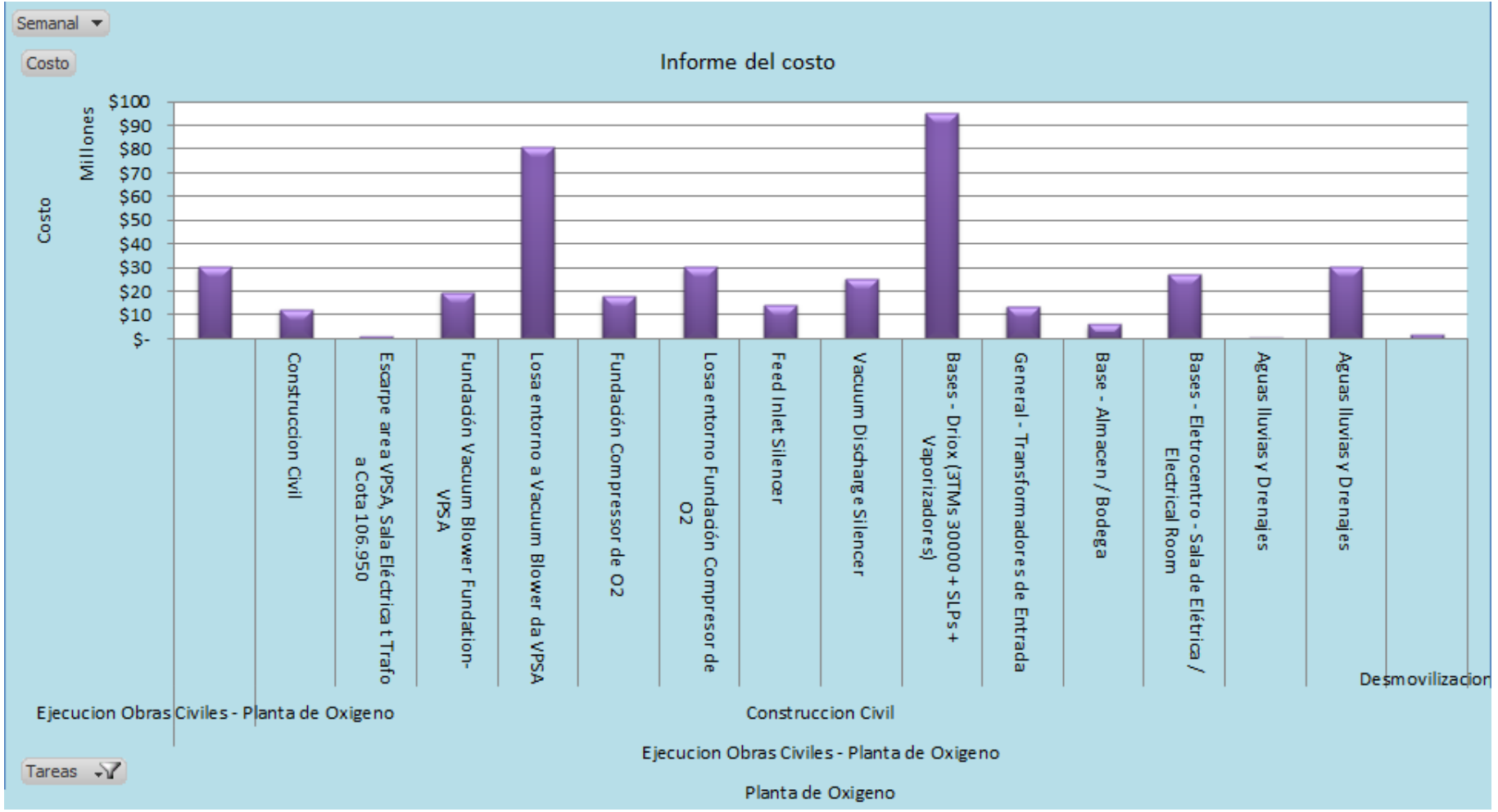

Figura 6: Informe de costo previo por partida, software Project

Tabla 5: Planilla de costos por partidas, software Project

\begin{tabular}{|c|c|c|c|c|}
\hline Semanal & Todos & & & \\
\hline \multicolumn{5}{|l|}{ Costo } \\
\hline Tarea & Tarea 1 & Tarea 2 & Tarea 3 & Total \\
\hline \multirow[t]{18}{*}{ Planta de Oxigeno } & $\begin{array}{l}\text { Ejecucion Obras Civiles - } \\
\text { Planta de Oxigeno }\end{array}$ & $\begin{array}{l}\text { Ejecucion Obras Civiles } \\
\text { - Planta de Oxigeno }\end{array}$ & & $\$ 30.670 .560$ \\
\hline & & Construccion Civil & Construccion Civil & $\$ 12.531 .584$ \\
\hline & & & $\begin{array}{l}\text { Escarpe area VPSA, Sala Eléctrica t Trafo a Cota } \\
106.950\end{array}$ & $\$ 1.233 .600$ \\
\hline & & & Fundación Vacuum Blower Fundation- VPSA & $\$ 19.432 .095$ \\
\hline & & & Losa entorno a Vacuum Blower da VPSA & $\$ 80.763 .781$ \\
\hline & & & Fundación Compressor de O2 & $\$ 17.757 .382$ \\
\hline & & & Losa entorno Fundación Compresor de $\mathrm{O} 2$ & $\$ 30.142 .919$ \\
\hline & & & Feed Inlet Silencer & $\$ 14.439 .798$ \\
\hline & & & Vacuum Discharge Silencer & $\$ 25.429 .364$ \\
\hline & & & $\begin{array}{l}\text { Bases - Driox (3TMs } 30000 \text { + SLPs + Vaporiza- } \\
\text { dores) }\end{array}$ & $\$ 95.067 .828$ \\
\hline & & & General - Transformadores de Entrada & $\$ 13.640 .109$ \\
\hline & & & Base - Almacen / Bodega & $\$ 6.113 .940$ \\
\hline & & & $\begin{array}{l}\text { Bases - Eletrocentro - Sala de Elétrica / Electri- } \\
\text { cal Room }\end{array}$ & $\$ 27.374 .118$ \\
\hline & & & Aguas lluvias y Drenajes & $\$ 824.000$ \\
\hline & & & Aguas lluvias y Drenajes & $\$ 30.296 .100$ \\
\hline & & Total Construccion Civil & & $\$ 375.046 .618$ \\
\hline & & Desmovilizacion & & $\$ 2.056 .000$ \\
\hline & $\begin{array}{l}\text { Total Ejecucion Obras Civiles } \\
\text { - Planta de Oxigeno }\end{array}$ & & & $\$ 407.773 .178$ \\
\hline $\begin{array}{l}\text { Total Planta de } \\
\text { Oxigeno }\end{array}$ & & & & $\$ 407.773 .178$ \\
\hline Total general & & & & $\$ 407.773 .178$ \\
\hline
\end{tabular}


en la fase de construcción de la obra, ya que, gracias a las simulaciones y las actualizaciones, se podrá tener la certeza de los avances de obra y costos durante todo el proyecto. Será responsabilidad del depto. de adquisiciones la entrega de las cotizaciones de materiales para la obra y responsabilidad del depto. de recursos humanos, el fijar los costos de sueldos. Ambos deberán hacer entrega de esta información al depto. de estudios de propuestas, el que realizará la inclusión de los recursos a la planificación y posteriormente entrega para aprobación de Gerencia. La planilla de recursos generada para la metodología, se observa en la Tabla 4. Además, se debe extraer desde Project a Excel, a planilla de costo por actividad, a través de los informes visuales de costos, como la mostrada en la Figura 6, bajo la información mostrada en la Tabla 5.

La productividad del proyecto se verá reflejada una vez iniciada la obra, ya que aquí se podrá determinar si los tiempos y/o rendimientos asignados a cada actividad, fueron estimados correctamente. Éstos deberán ser ajustados a la realidad de la obra, lo que tendrá un impacto directo en los costos. En la fase de estudio de propuesta, será responsabilidad del depto. de estudio de propuesta la estimación del rendimiento por actividad y responsabilidad del ingeniero planificador de obra, la actualización de estos rendimientos una vez iniciada la obra.

Con el fin de mejorar la estimación de tiempo por actividad se deberá extraer el rendimiento de la fuerza laboral por partida, con el fin de crear una base de datos con los tiempos de cada partida por especialidad. Esto es extraído de la planificación mediante los avances de obra identificando los rendimientos reales por actividad. Se deberá hacer por medio de informes visuales entregados por el software Project, como la mostrada en la Tabla 6. Al establecer el rendimiento de las partidas, se podrá automatizar de mejor forma el proceso de planificación.

\section{Vinculación y visualizador}

La vinculación de la información es un pilar fundamental dentro de la metodología, dado que es en este punto donde se entrelazan todas las especialidades y departamentos incluídos en el proyecto. También es donde se detectan los errores a priori, dando solución previa al inicio de faena. Es por esto que el responsable (coordinador BIM), deberá tener vasta experiencia y capacidades, a la hora de trabajar con distintos formatos y tener conocimiento y experiencia con el área de la construcción industrial para la detección

Tabla 6: Planilla de HH por partida, software Project

\begin{tabular}{|c|c|c|c|c|}
\hline Semanal & Todos & & & \\
\hline & & & & Datos \\
\hline Tarea & Tarea 1 & Tarea 2 & Tarea 3 & Trabajo \\
\hline \multirow[t]{14}{*}{$\begin{array}{l}\text { Planta de } \\
\text { Oxigeno }\end{array}$} & $\begin{array}{l}\text { Ejecucion Obras Civiles - } \\
\text { Planta de Oxigeno }\end{array}$ & $\begin{array}{l}\text { Ejecucion Obras Civiles } \\
\text { - Planta de Oxigeno }\end{array}$ & & 4305 \\
\hline & & Construccion Civil & Construccion Civil & 1843 \\
\hline & & & Escarpe area VPSA, Sala Eléctrica t Trafo a Cota 106.950 & 144 \\
\hline & & & Fundación Vacuum Blower Fundation- VPSA & 7071 \\
\hline & & & Fundación Compressor de $\mathrm{O} 2$ & 5095 \\
\hline & & & Losa entorno Fundación Compresor de $\mathrm{O} 2$ & 8891 \\
\hline & & & Feed Inlet Silencer & 3513 \\
\hline & & & Vacuum Discharge Silencer & 6752 \\
\hline & & & Bases - Driox (3TMs $30000+$ SLPs + Vaporizadores) & 39576 \\
\hline & & & General - Transformadores de Entrada & 3856 \\
\hline & & & Aguas lluvias y Drenajes & 1983 \\
\hline & & Total Construccion Civil & & 112881 \\
\hline & & Desmovilizacion & & 240 \\
\hline & $\begin{array}{l}\text { Total Ejecucion Obras Ci- } \\
\text { viles - Planta de Oxigeno }\end{array}$ & & & 117426 \\
\hline $\begin{array}{l}\text { Total Planta } \\
\text { de Oxigeno }\end{array}$ & & & & 117426 \\
\hline Total general & & & & 117426 \\
\hline
\end{tabular}


Bustamante, G., Ochoa, J. y González, F. (2021). Propuesta de implementación de la metodología BIM 5D para obras de cimentaciones industriales en la Planta de Oxígeno de Arauco. Obras y Proyectos 30, 74-90

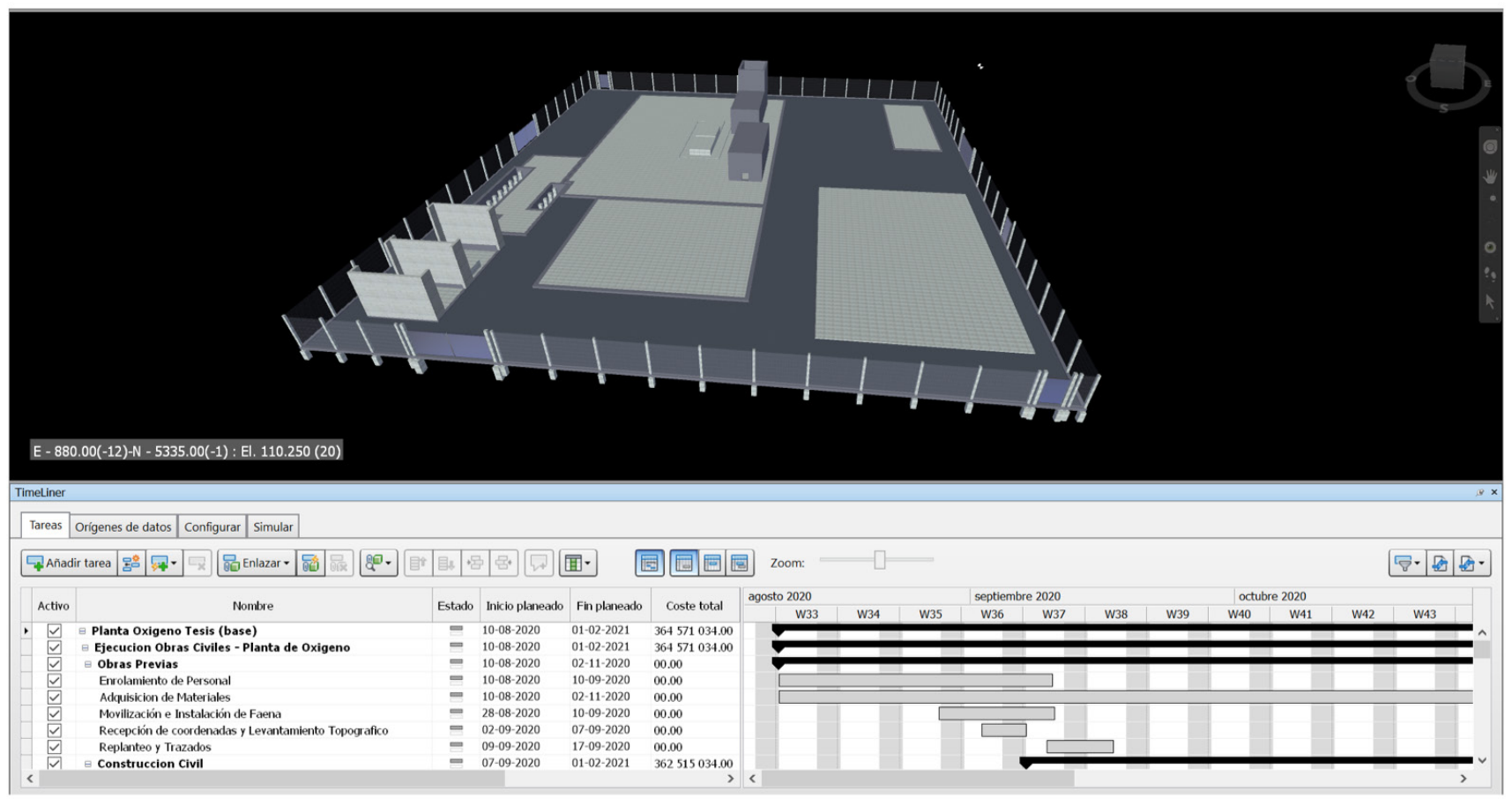

Figura 7: Vinculación de la Planificación con un modelo 3D, software Navisworks

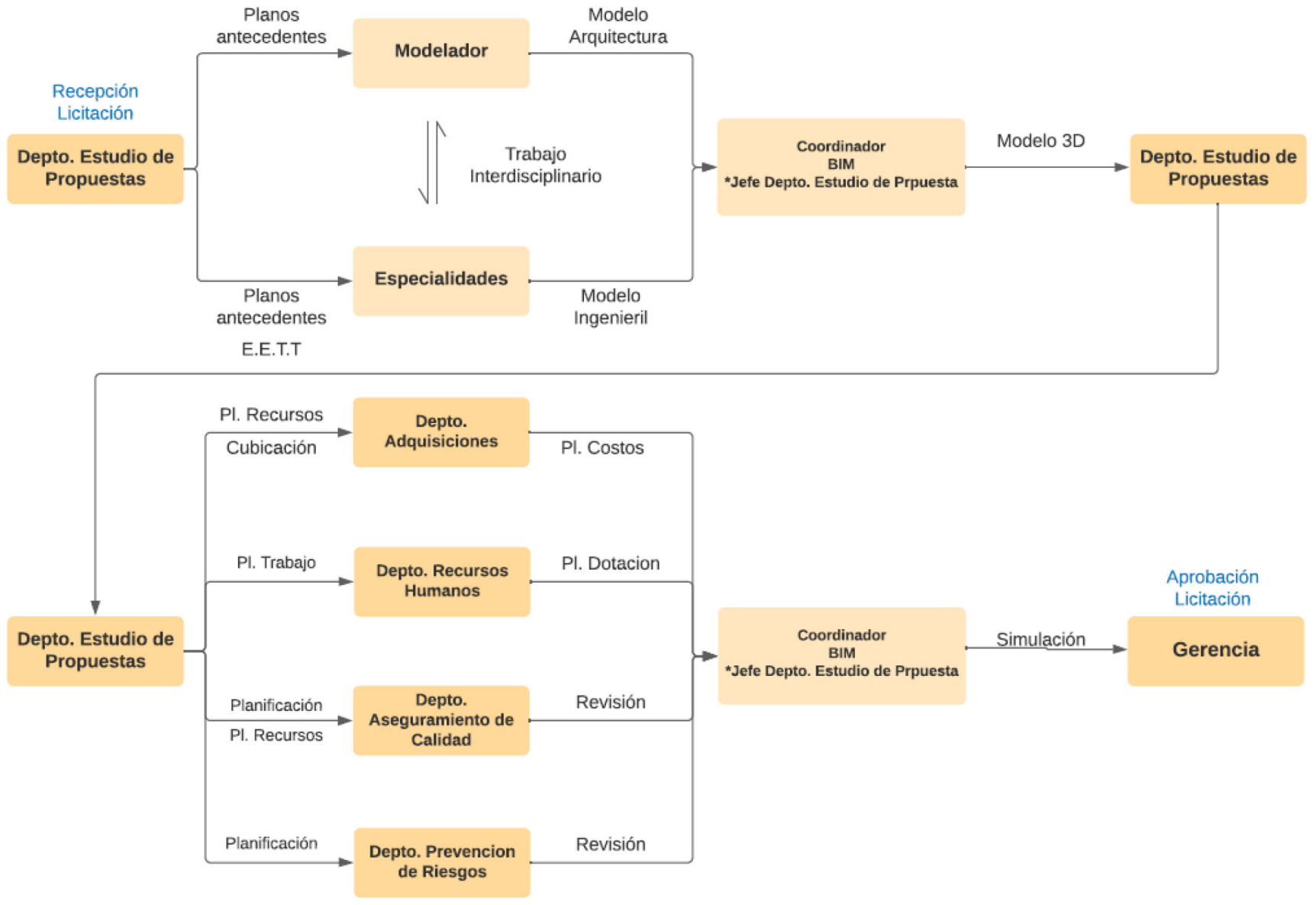

Figura 8: Esquema de gestión de la información en fase de licitación 
de irregularidades o errores de estimación dentro del estudio. El encargado dentro de la empresa será el Jefe de depto. de estudio de propuesta, el que deberá vincular la información para luego simular el proyecto.

Para la vinculación de las especialidades se utiliza el software REVIT, en el cual se realizará la superposición de modelos de las distintas especialidades, obteniendo el modelo 3D definitivo. Esto es responsabilidad del depto. de estudio de propuesta bajo el rol de Modelación BIM. El modelo será enlazado a la planificación mediante el software NAVISWORKS, el cual además de entregar una visualización 3D del proyecto, podrá crear la simulación del proyecto incluyendo costos y tiempo. La vinculación extraída del programa para el proyecto es mostrada en la Figura 7.

\section{Gestión de la información}

El manejo de información es responsabilidad de muchos participantes de esta metodología. Es vital tener en claro el papel que cumple cada participante dentro de la empresa para cada rol, de modo de facilitar el flujo de información a la hora de una licitación. Dada la gran cantidad de información dentro de un proyecto y la necesidad de extracción de información en las diferentes etapas del proyecto, se incluye un esquema del flujo de información teniendo en cuenta las planillas, modelos y datos dentro de la metodología. Las planillas, modelos y/o documentos deberán ser generados por los diferentes participantes, cuya información debe ser dirigida a los distintos departamentos como se ve en la Figura 8 (modelo 3D arquitectónico, modelo 3D ingenieril, cubicación, planilla de recursos, planilla de costos, planilla de trabajo $\mathrm{HH}$, planilla de cotizaciones, vinculación, simulación planificación (c/ recursos) y carta Gantt).

Para efectos de aprobación en cada etapa del proyecto, el Coordinador BIM debe incluir a la gerencia en cada etapa de la metodología, ya sea Gerencia General y/o Gerencia de Operaciones, evitando la falta de comunicación entre el proyecto y Oficina Central. En la fase de construcción, es necesario actualizar la información entregada en la fase de licitación, lo cual será responsabilidad de la oficina técnica de obra. Dicha información estará basada en los avances de obra. Mediante esta información se actualizarán las planillas de costo, trabajo, dotación e incluso modelo 3D. El administrador de contrato en la fase de construcción, cumple con el rol de coordinador. Deberá generar reportes de obra, informando las actualizaciones a gerencia. Además, a modo de perfeccionar la metodología, de forma interna, la oficina técnica aportará información de rendimientos y costos por partida, con el fin de ajustar la estimación. Este flujo de información es esquematizado en la Figura 9.

\section{Resultados \\ Roles y profesionales}

Los roles estipulados en Tabla 2, con la asignación de tareas, conduce a la necesidad de personal capacitado para el manejo de información BIM. El personal mínimo para el desarrollo de la metodología está establecido en la Tabla 7, según las responsabilidades por departamento, también muestra el personal actualmente por departamento, bajo el cargo de cada participante. Es indispensable la contratación de especialistas en BIM, para la coordinación de la información

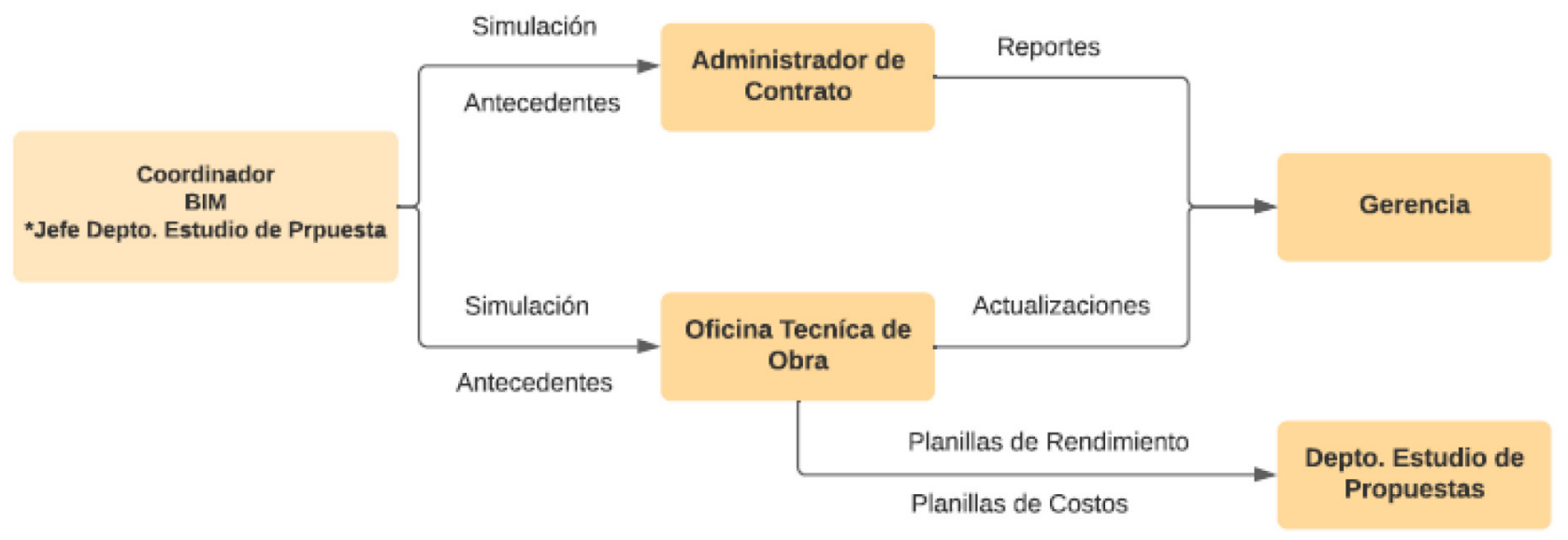

Figura 9: Esquema de gestión de la información en la empresa, fase de construcción 
Tabla 7: Personal en la empresa y personal necesario en la metodología

\begin{tabular}{|c|c|c|c|}
\hline Fase & Departamento & Personal Actual & $\begin{array}{l}\text { Personal } \\
\text { Necesario para } \\
\text { metodología }\end{array}$ \\
\hline \multirow{12}{*}{ Licitación } & \multirow[b]{2}{*}{ Gerencia } & Gerente General & \multirow[b]{2}{*}{ Dirección BIM } \\
\hline & & $\begin{array}{l}\text { Gerente de } \\
\text { Operaciones }\end{array}$ & \\
\hline & \multirow{4}{*}{$\begin{array}{l}\text { Estudio de } \\
\text { Propuestas }\end{array}$} & $\begin{array}{l}\text { Jefe de } \\
\text { Departamento }\end{array}$ & $\begin{array}{l}\text { Coordinador } \\
\text { BIM }\end{array}$ \\
\hline & & $\begin{array}{l}\text { Ingeniero de } \\
\text { Estudios (1) } \\
\end{array}$ & $\begin{array}{l}\text { Especialista/ } \\
\text { Planificador } \\
\end{array}$ \\
\hline & & $\begin{array}{l}\text { Ingeniero de } \\
\text { Estudios (2) }\end{array}$ & $\begin{array}{l}\text { Especialista/ } \\
\text { Planificador }\end{array}$ \\
\hline & & & Modelador \\
\hline & \multirow{2}{*}{ Adquisiciones } & $\begin{array}{l}\text { Jefe de Departa- } \\
\text { mento }\end{array}$ & Gestión BIM \\
\hline & & $\begin{array}{l}\text { Gestión de } \\
\text { Compras }\end{array}$ & Gestión BIM \\
\hline & \multirow{2}{*}{$\begin{array}{l}\text { Recursos } \\
\text { humanos }\end{array}$} & $\begin{array}{l}\text { Jefe de } \\
\text { Departamento }\end{array}$ & Gestión BIM \\
\hline & & Administrativo & Gestión BIM \\
\hline & $\begin{array}{l}\text { Aseguramiento } \\
\text { de Calidad }\end{array}$ & $\begin{array}{l}\text { Jefe de } \\
\text { Departamento }\end{array}$ & $\begin{array}{l}\text { Gestión BIM/ } \\
\text { Revisor BIM }\end{array}$ \\
\hline & $\begin{array}{l}\text { Prevención de } \\
\text { Riesgos }\end{array}$ & $\begin{array}{l}\text { Jefe de } \\
\text { Departamento }\end{array}$ & $\begin{array}{l}\text { Gestión BIM/ } \\
\text { Revisor BIM }\end{array}$ \\
\hline \multirow{5}{*}{ Construcción } & $\begin{array}{l}\text { Administrador } \\
\text { de contrato }\end{array}$ & $\begin{array}{l}\text { Ingeniero } \\
\text { Administrador }\end{array}$ & $\begin{array}{l}\text { Coordinador } \\
\text { BIM }\end{array}$ \\
\hline & \multirow{4}{*}{$\begin{array}{l}\text { Oficina } \\
\text { Técnica }\end{array}$} & $\begin{array}{l}\text { Ingeniero } \\
\text { Planificador }\end{array}$ & $\begin{array}{l}\text { Modelador/ } \\
\text { Gestión BIM }\end{array}$ \\
\hline & & $\begin{array}{l}\text { Aseguramiento de } \\
\text { Calidad }\end{array}$ & $\begin{array}{l}\text { Gestión BIM/ } \\
\text { Revisor BIM }\end{array}$ \\
\hline & & Prevención & $\begin{array}{l}\text { Gestión BIM/ } \\
\text { Revisor BIM }\end{array}$ \\
\hline & & Administrativo & Gestión BIM \\
\hline
\end{tabular}

BIM en la oficina central, como en la oficina técnica. Además, la capacitación del personal actual para la actualización de la metodología utilizada hoy en día por la empresa.

De la Tabla 7 se desprende que no se requiere contratar más personal, solo hay que instruir al existente. Si es necesario implementar el rol de modelador que no existe en la dotación de personal actual.

\section{Análisis de metodología}

Al momento de aplicar la metodología en la obra Construcción de una Planta de Oxígeno y al ser partícipe del proyecto, se detectaron diversos problemas en gestión de información, en el diseño del proyecto, por lo que se debió hacer consultas desde la oficina técnica de obra al mandante en la fase de construcción. La comunicación entre las partes del proyecto juega una parte importante dentro de los tiempos de las actividades, ya que no pueden ser finalizadas, sin previa aprobación de las modificaciones o consultas realizadas, con una estimación de resolución de la consulta de 3 días hábiles. Esto debido al promedio de respuesta percibido en obra. Además, se identifican errores en la recepción de suministros, lo que llevó a tomar decisiones en terreno, elevando el costo de partidas. Esto podría haber sido evitado con el uso de la metodología, ya que estas consultas podrían ser resueltas mediante la interpretación del modelo, como también la actualización de la metodología y nuevas simulaciones, las que ayudarían a la reorganización de la planificación y tareas críticas dentro del proyecto. Los principales defectos encontrados dentro del desarrollo del proyecto fueron: a) errores de niveles dentro de los planos de antecedentes, b) mal dimensionado de enfierraduras, c) toma de decisiones apresuradas en terreno, dada la interpretación de planos y e) poca información de los detalles de los distintos elementos.

Para establecer los beneficios, se realiza la comparación de las metodologías en las cinco dimensiones, identificando las diferencias encontradas en cada dimensión para cada partida analizada. Es importante resaltar que los resultados de la nueva metodología, fueron obtenidos al aplicarla en la fase de construcción, es decir, cuando el proyecto ya está en marcha. Se prevé que, al aplicar la metodología desde la etapa de estudio de propuesta, los beneficios sean mayores.

\section{Partidas analizadas}

Tras la realización del proyecto con la metodología BIM y teniendo la información proveniente de obra, se ve que la metodología implementada hoy en día por la empresa genera beneficios importantes. Se busca establecer las diferencias entre ambas metodologías, por lo que se identifican las partidas con mayor diferencia de tiempo y costo. Las partidas seleccionadas para el análisis en las cinco dimensiones, son las mostradas en la Tabla 8.

Tabla 8: Partidas analizadas

\begin{tabular}{|c|c|}
\hline Elemento & Actividad \\
\hline \multirow{2}{*}{ Fundación Driox } & Excavación \\
\cline { 2 - 2 } & Enfierradura 10 \\
\cline { 2 - 2 } & Hormigonado 13 \\
\hline \multirow{2}{*}{ Fundación Losa VPSA } & Excavación \\
\cline { 2 - 2 } & Hormigonado2, 3, 4 \\
\hline
\end{tabular}

Como se aprecia, las cinco partidas analizadas son agrupadas en dos cimentaciones. A continuación, se analizan las partidas según su incidencia en cada dimensión. Para la visualización de los elementos a analizar, se aíslan en el modelo 3D, extrayendo visualizaciones como las mostradas en las Figuras 10 y 11. 


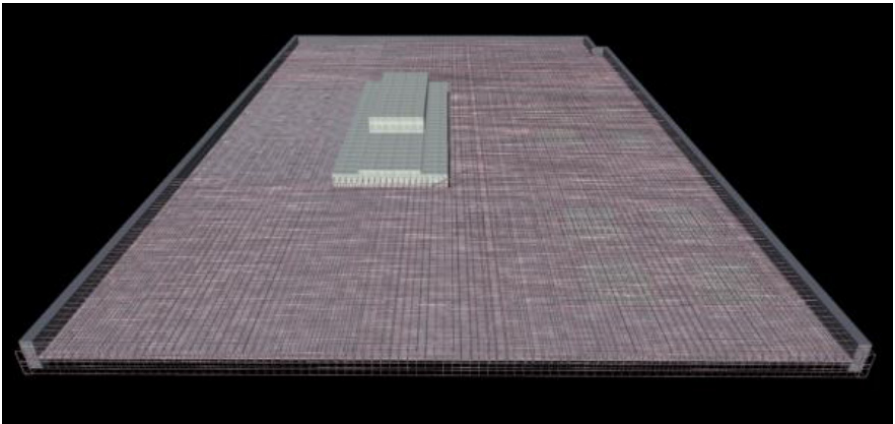

Figura 10: Fundación losa VPSA, software Navisworks

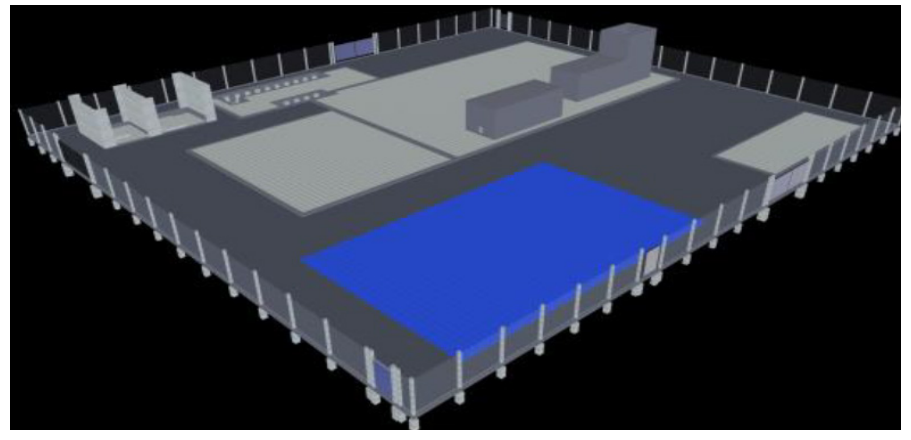

Figura 11: Fundación DRIOX, software Navisworks

Tabla 9: Tabla de recursos para la planificación de la empresa y BIM, software Project

\begin{tabular}{|c|c|c|c|c|c|c|c|c|c|c|}
\hline \multirow{2}{*}{$\begin{array}{c}\text { Cimenta- } \\
\text { ción }\end{array}$} & \multirow[b]{2}{*}{ Actividad } & \multirow[b]{2}{*}{ Recursos } & \multicolumn{3}{|c|}{ CAVCO } & \multicolumn{3}{|c|}{ BIM } & \multirow{2}{*}{$\begin{array}{l}\text { Difenren- } \\
\text { cia }\end{array}$} & \multirow[b]{2}{*}{ Unidad } \\
\hline & & & $\begin{array}{l}\text { Tra- } \\
\text { bajo }\end{array}$ & Material & Unidad & Trabajo & $\begin{array}{l}\text { Mate- } \\
\text { rial }\end{array}$ & Unidad & & \\
\hline \multirow{35}{*}{$\begin{array}{l}\text { Funda- } \\
\text { ción } \\
\text { DRIOX }\end{array}$} & \multirow{6}{*}{ Excavación } & Operador 1 & 152 & & horas & 152 & & horas & 0 & horas \\
\hline & & Maestro Civil $1^{\circ} 13$ & 152 & & horas & 152 & & horas & 0 & horas \\
\hline & & Maestro Civil $1^{\circ} 14$ & 152 & & horas & 152 & & horas & 0 & horas \\
\hline & & Maestro Civil $2^{\circ} 13$ & 152 & & horas & 152 & & horas & 0 & horas \\
\hline & & Maestro Civil $2^{\circ} 14$ & 152 & & horas & 152 & & horas & 0 & horas \\
\hline & & Retroescavadora & 152 & & horas & 152 & & horas & 0 & horas \\
\hline & \multirow{13}{*}{$\begin{array}{c}\text { Enfierradu- } \\
\text { ra10 }\end{array}$} & Maestro Civil $1^{\circ} 11$ & 90,6 & & horas & 90,6 & & horas & 0 & horas \\
\hline & & Maestro Civil $1^{\circ} 12$ & 90,6 & & horas & 90,6 & & horas & 0 & horas \\
\hline & & Maestro Civil $2^{\circ} 11$ & 90,6 & & horas & 90,6 & & horas & 0 & horas \\
\hline & & Maestro Civil $2^{\circ} 12$ & 90,6 & & horas & 90,6 & & horas & 0 & horas \\
\hline & & Enfierrador7 & 90,6 & & horas & 90,6 & & horas & 0 & horas \\
\hline & & Enfierrador8 & 90,6 & & horas & 90,6 & & horas & 0 & horas \\
\hline & & Enfierrador9 & 90,6 & & horas & 90,6 & & horas & 0 & horas \\
\hline & & Fierro Estriado $n^{\circ} 10$ & & 210,4 & $\mathrm{~kg}$ & & 210,4 & $\mathrm{~kg}$ & 0,0 & $\mathrm{~kg}$ \\
\hline & & Fierro Estriado $n^{\circ} 12$ & & 623,4 & $\mathrm{~kg}$ & & 623,4 & $\mathrm{~kg}$ & 0,0 & $\mathrm{~kg}$ \\
\hline & & Fierro Estriado ${ }^{\circ} 16$ & & 2150,0 & $\mathrm{~kg}$ & & 0,0 & $\mathrm{~kg}$ & 2150,0 & $\mathrm{~kg}$ \\
\hline & & Fierro Estriado $n^{\circ} 25$ & & 3085,2 & $\mathrm{~kg}$ & & 3085,2 & $\mathrm{~kg}$ & 0,0 & $\mathrm{~kg}$ \\
\hline & & Fierro Estriado $n^{\circ} 28$ & & 30532,6 & $\mathrm{~kg}$ & & 30532,6 & $\mathrm{~kg}$ & 0,0 & $\mathrm{~kg}$ \\
\hline & & Alambre Negro & & 1178,0 & $\mathrm{~kg}$ & & 1178,0 & $\mathrm{~kg}$ & 0,0 & $\mathrm{~kg}$ \\
\hline & \multirow{16}{*}{$\begin{array}{l}\text { Hormigona- } \\
\text { do13 }\end{array}$} & Maestro Civil $1^{\circ} 1$ & 8 & & horas & 8 & & horas & 0 & horas \\
\hline & & Maestro Civil $1^{\circ} 2$ & 8 & & horas & 8 & & horas & 0 & horas \\
\hline & & Maestro Civil $2^{\circ} 1$ & 8 & & horas & 8 & & horas & 0 & horas \\
\hline & & Maestro Civil $2^{\circ} 2$ & 8 & & horas & 8 & & horas & 0 & horas \\
\hline & & Maestro Civil $3^{\circ} 1$ & 8 & & horas & 8 & & horas & 0 & horas \\
\hline & & Maestro Civil $3^{\circ} 2$ & 8 & & horas & 8 & & horas & 0 & horas \\
\hline & & Maestro Civil $4^{\circ} 1$ & 8 & & horas & 8 & & horas & 0 & horas \\
\hline & & Maestro Civil $4^{\circ} 2$ & 8 & & horas & 8 & & horas & 0 & horas \\
\hline & & Maestro Civil $5^{\circ} 1$ & 8 & & horas & 8 & & horas & 0 & horas \\
\hline & & Maestro Civil $5^{\circ} 2$ & 8 & & horas & 8 & & horas & 0 & horas \\
\hline & & Sonda Vibradora 1 & 8 & & horas & 8 & & horas & 0 & horas \\
\hline & & Sonda Vibradora 2 & 8 & & horas & 8 & & horas & 0 & horas \\
\hline & & Sonda Vibradora 3 & 8 & & horas & 8 & & horas & 0 & horas \\
\hline & & $\begin{array}{l}\text { Servicio de Bombeo Hor- } \\
\text { migón }\end{array}$ & & 348 & $\mathrm{~m}^{3}$ & & 289,6 & $\mathrm{~m}^{3}$ & 58,4 & $\mathrm{~m}^{3}$ \\
\hline & & Laboratorio Hormigón & & 3 & sets & & 3 & sets & 0 & sets \\
\hline & & Hormigón H30 & & 348 & $\mathrm{~m}^{3}$ & & 289,6 & $\mathrm{~m}^{3}$ & 58,4 & $\mathrm{~m}^{3}$ \\
\hline
\end{tabular}




\section{Análisis 3D}

En base al modelo 3D, se realiza la cubicación del proyecto, mediante la extracción de forma y materialidad de los distintos elementos. La cubicación de las partidas, se muestran en la Tabla 9. Se observa una diferencia de cubicación en los recursos de hormigón $\left(58.4 \mathrm{~m}^{3}\right)$ y fierro estriado $\mathrm{n}^{\circ} 16(2150 \mathrm{~kg})$. Esto debido a diferencia de cotas, entre ambos modelos. Dicha diferencia se establece por la interpretación de los planos de la obra DF-25075PRX-11-003, DF-25075-PRX-11-004 y DF-25075PRX-14-001, los cuales contradicen el nivel del hormigón, con una diferencia en promedio de $7.5 \mathrm{~cm}$ en toda la losa. En el caso de la metodología BIM, al tratarse de un solo modelo, la interpretación es única, por lo que se evitaría esta falla en el proceso de construcción. La diferencia en el fierro estriado se debió a que al interpretarse mal el espesor de la losa, se construyeron los fierros espaciadores de malla (conocidos en faena como ranas) de una longitud tal que no era. Luego se tuvieron que rehacer todos estos espaciadores, lo que arrojó una pérdida de material como la que se indica.

La situación es que los fierros separadores de las mallas inferior y superior no estaban indicadas en el proyecto estructural. Cuando la persona que las consideró las dimensionó y se ejecutarton se hicieron de la forma correcta. Sin embargo, cuando se trazó la losa en terreno otra persona indicó que la losa tenía un espesor diferente al de los planos y en consecuencia rechazó los separadores que se había hecho. Lo que conllevó a hacerlos de nuevo y la cantidad de fiero adicional que se tuvo que confeccionar fue de $2150 \mathrm{~kg}$. Si todo este proceso se hubiera realizado bajo la propuesta de este trabajo la situación se hubiera identificado de inmediato y no se debería haber incurrido en el gasto adicional de separadores y aumento de espesor de la losa.

\section{Análisis 4D}

En la cuarta dimensión, se analiza la planificación llevada a cabo en el proyecto comparando con la de la metodología BIM, se extraen de ambas planificaciones y se identifican las partidas a analizar. Esto se puede ver en la Tabla 10, para la metodología actual de la empresa y en la Tabla 11, para BIM. Al interpretar ambas tablas se identifica que mediante la metodología BIM el tiempo en la actividad de excavación es menor. Esto debido a que se debió comprobar la información de cotas. Si bien, en la metodología BIM las actividades de excavación se vieron reducidas en 3 días cada una, la duración la partida Fundación Losa VPSA, se vio disminuida en solo 1 día, ya que las otras actividades involucradas en la ejecución completa de la fundación formaban parte de la ruta crítica de la partida.

Tabla 10: Duración partidas con planificación de la empresa, software Project

\begin{tabular}{|l|l|l|l|}
\hline Nombre de tarea & Duración & Comienzo & Fin \\
\hline Fundación DRIOX & 66.6 días & mar 06-10-20 & mar $12-01-21$ \\
\hline Excavación & 13.89 días & mar $06-10-20$ & mar $27-10-20$ \\
\hline Enfierradura10 & 10.07 días & mié $18-11-20$ & mié $02-12-20$ \\
\hline Hormigonado13 & 0.89 días & jue $24-12-20$ & lun $28-12-20$ \\
\hline Fundación Losa VPSA & 32.26 días & lun 21-09-20 & mar $10-11-20$ \\
\hline Excavación & 9.49 días & lun 21-09-20 & mié $07-10-20$ \\
\hline Hormigonado2 & 0.89 días & mar $03-11-20$ & mié $04-11-20$ \\
\hline Hormigonado3 & 0.89 días & jue $12-11-20$ & vie $13-11-20$ \\
\hline Hormigonado4 & 0.89 días & vie $04-12-20$ & lun 07-12-20 \\
\hline
\end{tabular}

Tabla 11: Duración de partidas con planificación BIM, software Project

\begin{tabular}{|l|l|l|l|}
\hline Nombre de tarea & Duración & Comienzo & Fin \\
\hline Fundación DRIOX & 66.6 días & mar 06-10-20 & mar 12-01-21 \\
\hline Excavación & 13.89 días & mar 06-10-20 & mar 27-10-20 \\
\hline Enfierradura10 & 10.07 días & mié 18-11-20 & mié 02-12-20 \\
\hline Hormigonado13 & 0.89 días & jue 24-12-20 & lun 28-12-20 \\
\hline Fundación Losa VPSA & 34.37 días & lun 21-09-20 & lun 09-11-20 \\
\hline Excavación & 6.49 días & lun 21-09-20 & vier 02-10-20 \\
\hline Hormigonado2 & 0.89 días & lun 02-11-20 & mar 03-11-20 \\
\hline Hormigonado3 & 0.89 días & lun 09-11-20 & mar 10-11-20 \\
\hline Hormigonado4 & 0.89 días & mar 01-12-20 & mié 02-12-20 \\
\hline
\end{tabular}

Tabla 12: Costos de planificación de la empresa y planificación BIM, software Project

\begin{tabular}{|l|l|l|}
\hline \multicolumn{1}{|c|}{ Nombre de tarea } & \multicolumn{2}{c|}{ Costo } \\
\hline Fundación losa VPSA & Empresa & BIM \\
\hline Excavación & $\$ 4.387 .504$ & $\$ 3.002 .274$ \\
\hline Hormigonado2 & $\$ 15.579 .000$ & $\$ 13.821 .000$ \\
\hline Hormigonado3 & $\$ 11.229 .000$ & $\$ 10.054 .500$ \\
\hline Hormigonado4 & $\$ 14.781 .500$ & $\$ 13.230 .00$ \\
\hline Fundación DRIOX & Empresa & BIM \\
\hline Excavación & $\$ 7.812 .800$ & $\$ 6.425 .214$ \\
\hline Enfierradura10 & $\$ 27.942 .160$ & $\$ 26.544 .660$ \\
\hline Hormigonado13 & $\$ 51.141 .200$ & $\$ 42.673 .850$ \\
\hline
\end{tabular}

\section{Análisis 5D}

Para las partidas analizadas se cuantificó el costo asociado a las actividades, con la disminución de tiempo y recurso para 
cada actividad, dada la nueva planificación. En la Tabla 12, se muestran los costos asociados a la planificación con la cual se construyó y los costos asociados a la planificación BIM. Al realizar la comparación de los costos de ambas planificaciones, como se muestra en la Tabla 13, se observa que la diferencia de costo es de $\$ 18.503 .811$. Esto al aplicar la metodología en la fase de construcción.

Tabla 13: Comparación costos por partida en planificación de la empresa y planificación BIM

\begin{tabular}{|c|c|c|c|c|}
\hline \multirow{2}{*}{ Elemento } & \multirow{2}{*}{ Actividad } & \multicolumn{2}{|c|}{ Planificacion } & \multirow{2}{*}{ Diferencia } \\
\hline & & BIM & Empresa & \\
\hline \multirow{3}{*}{$\begin{array}{l}\text { Fundación } \\
\text { Driox }\end{array}$} & $\begin{array}{c}\text { Retraso } \\
\text { en partida }\end{array}$ & $\$ 6.425 .514$ & $\$ 7.812 .800$ & $\$ 1.387 .286$ \\
\hline & $\begin{array}{l}\text { Enfierra- } \\
\text { dura }\end{array}$ & $\$ 26.544 .660$ & $\$ 27.942 .160$ & $\$ 1.377 .500$ \\
\hline & $\begin{array}{l}\text { Hormigo- } \\
\text { nado }\end{array}$ & $\$ 42.673 .850$ & $\$ 51.141 .200$ & $\$ 8.467 .350$ \\
\hline \multirow{2}{*}{$\begin{array}{l}\text { Fundación } \\
\text { Losa VPSA }\end{array}$} & $\begin{array}{c}\text { Retraso } \\
\text { en partida }\end{array}$ & $\$ 3.002 .274$ & $\$ 4.387 .504$ & $\$ 1.385 .230$ \\
\hline & $\begin{array}{l}\text { Hormigo- } \\
\text { nado }\end{array}$ & $\$ 37.105 .500$ & $\$ 41.589 .500$ & $\$ 4.484 .000$ \\
\hline Total Obra & $\begin{array}{c}\text { Duración } \\
\text { Total }\end{array}$ & $\$ 390.914 .067$ & $\$ 409.417 .878$ & $\$ 18.503 .811$ \\
\hline
\end{tabular}

\section{Costos metodología}

El uso de la metodología también tiene costos asociados, la capacitación del personal y necesidad de más personal, debido a lo establecido en el Tabla 7. Estos costos fueron cuantificados, según el mercado actual. Estos se muestran en la Tabla 14. Cabe destacar que en este costo se considera la participación del personal actualmente contratado, lo que a su vez también tiene un costo de capacitación. El costo del coordinador de estudio, modelador y capacitación, será cubierto por más de un proyecto, en los que podrán participar paralelamente. No así, el coordinador BIM en el proyecto, ya que el deberá ser contratado por la duración total del proyecto, es quien deberá actualizar la información del modelo, planificación y costos. No obstante, al ser participantes necesarios para la metodología, se considera su sueldo total.

Tabla 14: Estimación costos para metodología BIM en la empresa

\begin{tabular}{|l|l|l|}
\hline \multicolumn{1}{|c|}{ Personal } & \multicolumn{1}{c|}{ Rol } & \multicolumn{1}{c|}{ Costo } \\
\hline Modelador BIM & Modelador & $\$ 800.000$ \\
\hline $\begin{array}{l}\text { Coordinador BIM } \\
\text { Estudio }\end{array}$ & Especialista/Modelador & $\$ 1.900 .000$ \\
\hline $\begin{array}{l}\text { Coordinador BIM } \\
\text { Terreno }\end{array}$ & Especialista/Modelador & $\$ 2.200 .000$ \\
\hline Capacitaciones & \multicolumn{1}{|c|}{ - } & $\$ 3.000 .000$ \\
\hline \multicolumn{2}{|c|}{ Total } & $\$ 7.900 .000$ \\
\hline
\end{tabular}

Los costos mencionados en la Tabla 14, son los salarios promedio para los puestos correspondientes. Además, se considera una suma alzada de la capacitación del personal contratado actualmente para estudio de propuestas, obteniendo un costo total de \$7.900.000. Sin embargo, tanto modelador, como Coordinador de estudio, participan del proyecto solo en la fase de diseño, por lo que se considera como un costo fijo, al igual que las capacitaciones, no así el coordinador de terreno cuyo costo es mensual. En el caso presentado los profesionales existen, pero no desarrollando la metodología BIM. Lo que se sugiere es capacitarlos en esta metodología. Los costos de capacitación y contratación, son establecidos en base al requerimiento de la obra, la dotación de profesionales será proporcional a la información necesaria para el proyecto.

\section{Conclusiones}

Al realizar la fase de construcción con la metodología BIM, se muestra un ahorro económico de $\$ 5.303 .811$, siendo esto el $1.3 \%$ del costo total del proyecto, incluyéndose el costo del coordinador BIM de terreno por la etapa de construcción del proyecto. Tras la nueva planificacion, las actividades de excavación analizadas tienen un término anticipado en 1 día hábil. Luego de los términos anticipados en las partidas analizadas, en la nueva planificación se observa una disminución de 3 días hábiles. Se comprueba que se cumplen todos los beneficios establecidos previamente a la utilización de la metodología BIM, tanto económica, como uso de la información.

Es necesario el uso de los softwares REVIT, NAVISWORKS y AUTOCAD para el desarrollo de la metodología BIM y PROJECT para una buena planificación, para el proceso de licitación y construcción. La implementación de la metodología se debe hacer desde el inicio de la fase de licitación o incluso en la fase de diseño. Es de vital de importancia para el flujo de información. Se verifica que se cumplen todos los beneficios establecidos previamente a la utilización de la metodología BIM.

Innegablemente, aplicar una nueva metodología en cualquier organización es un proceso lento y de alto costo de recursos. Sin embargo, la evolución de la industria de la construcción, obliga a actualizar la forma de trabajo. La utilización de la metodología se hace necesaria para obras de gran envergadura, debido al alto flujo de información para realizar el proyecto. Si bien es cierto, el uso de 
la metodología BIM significa mayor organización, es imprescindible actualizar la forma de trabajo, de modo de crear nuevos estándares, con respecto a las nuevas tecnologías a implementar en el área, estando a la vanguardia del rubro. Se prevé que aumente la eficiencia de las partidas al aplicar la metodología descrita, al mejorar la gestión de información entre los participantes, evitando errores y toma apresurada de decisiones en terreno. Así se podrá tener mayor control en el proyecto.

\section{Referencias}

AIA (2013). Building Information Modeling Protocol Form G202-2013. American Institute of Architects AIA (http://www. aia.org), Washington DC, USA

Aliaga Melo, G. (2012). Implementación y metodología para la elaboración de modelos BIM para su aplicación en proyectos industriales multidisciplinarios. Proyecto de título de Ingeniero Civil, Universidad de Chile, Santiago, Chile

CORFO (2015). Plan BIM Chile. Construye 2025. Santiago, Chile (https://planbim.cl)

Eastman, C., Teicholz, P., Sacks, R. and Liston, K. (2018). BIM handbook: A guide to building information modeling for owners, managers, designers, engineers and contractors. John Wiley \& Sons. USA

Forgues, D., Iordanova, I., Valdivesio, F. and Staub-French, S. (2012). Rethinking the cost estimating process through 5D BIM: A case study. Construction Research Congress 2012: Construction Challenges in a Flat World, West Lafayette, Indiana, USA, 778-786

Hasan, A.N. and Rasheed, S.M. (2019). The benefits of and challenges to implement 5D BIM in construction industry. Civil Engineering Journal 5(2), 412-421

ISO 19650-1 (2018). Organización de la información sobre trabajos de construcción - Gestión de la información en el uso del BIM, Parte 1: Conceptos y principios. Ginebra, Suiza
ISO 19650-2 (2018). Organización de la información sobre trabajos de construcción - Gestión de la información en el uso del BIM, Parte 2: Fase de diseño y producción de los inmuebles. Ginebra, Suiza

Kim, K.P., Ma, T., Baryah, A.S., Zhang, C. and Hui, K.M. (2016). Investigation of readiness for 4D and 5D BIM adoption in the Australian construction industry. Management Review: An International Journal 11(2), 43-64

Montilla, A. (2017). Las claves para implantar BIM en una empresa española. https://revistadigital.inesem.es/diseno-yartes-graficas/implantacionbim/

Pape, H. y Nazer, A. (2021). Determinantes de la innovación en empresas constructoras de la Región de Atacama, Chile. Obras y Proyectos 29, 80-92

Plan BIM (2017). Roles en BIM. Corporacion de Desarrollo Tecnológico CDT, CORFO. Santiago, Chile

PMG (2018). Diagnóstico de la situacion actual de formacion de capital humano en BIM en Chile. Consultora PMG, Santiago, Chile

Trejo Carvajal, N.A. (2018). Estudio de impacto del uso de la metodologia BIM en la planificación y control de proyectos de ingeniería y construcción. Proyecto de título de Ingeniero Civil, Universidad de Chile. Santiago, Chile

Stanley, R. and Thurnell, D. (2014). The benefits of, and barriers to, implementation of 5D BIM for quantity surveying in New Zealand. Australasian Journal of Construction Economics and Building 14(1), 105-117 